\title{
Thermal Conductivity Enhancement of Compact Heat Sinks Using Cellular Metals
}

\author{
T. Fiedler ${ }^{1, a}$, A. Öchsner, ${ }^{2, b}$, I.V. Belova ${ }^{3, c}$ and G.E. Murch ${ }^{3, d}$ \\ ${ }^{1}$ Department of Mechanical Engineering, University of Aveiro, 3810-193 Aveiro, Portugal \\ ${ }^{2}$ Department of Applied Mechanics, Faculty of Mechanical Engineering, Technical \\ University of Malaysia, 81310 UTM Skudai, Johor, Malaysia \\ ${ }^{3}$ Diffusion in Solids Group, Centre for Geotechnical and Materials Modelling, School of \\ Engineering, \\ The University of Newcastle, Callaghan, NSW 2308, Australia \\ a TFiedler@mec.ua.pt, ${ }^{b}$ Oechsner@fkm.utm.my \\ c Irina.Belova@newcastle.edu.au, d Graeme.Murch@newcastle.edu.au,
}

Keywords: Thermal conductivity, Cellular Metals, CVD Diamond, Lattice Monte Carlo

\begin{abstract}
In this paper, the increase of the effective thermal conductivity of paraffin based heat sinks is investigated by making use of cellular metallic matrixes with open cells which are introduced in the thermal low conductive paraffin wax. Lattice Monte Carlo analyses are conducted on different model geometries of such composites composed of a cellular matrixes and paraffin wax. The dependence of the effective thermal conductivity on the cell geometry and the metal foam matrix material is analysed. Furthermore, a diamond coating is simulated in order to estimate its influence on the effective thermal conductivity.
\end{abstract}

\section{Introduction}

Thermal management of electrical devices under cyclic temperature loading is important for proper operation of the devices and for thermal safety. Various phase change materials (PCM) can be used for thermal energy storage in the design of the passive heat sinks. A commonly chosen PCM is paraffin wax which is one of the most efficient materials for thermal energy storage at operating temperatures less than $100 \mathrm{C}$. However, paraffin exhibits a low thermal conductivity $(0.21 \mathrm{~W} /(\mathrm{m} \cdot \mathrm{K})$ [1]) at room temperature. This low thermal conductivity limits the performance of the thermal management systems, since thermal energy can only be slowly transferred into the heat sink. Accordingly, research (e.g. [2,3]) on the enhancement of the thermal conductivity using fins, honeycombs and porous media has been conducted. Mettawee and Assassa [1] enhanced the thermal conductivity of paraffin wax by adding aluminum powder. However, the embedded aluminium powder formed no interconnected matrix and the observed increase of conductivity was relatively low. Khateeb et al. [4,5] investigated the use of aluminium foam matrixes (cf. Fig. 2 a) in paraffin to improve the performance of the passive thermal management system of batteries. Hong and Herling [6] carried on this concept and investigated the effects of geometric parameters of aluminum foams on the performance of aluminum foam-PCM heat sinks. Both groups [4-6] observed a significant increase of the effective thermal conductivity $\lambda_{\text {eff }}$ of the aluminium-PCM composites. Closely related approaches $[3,7,8]$ addressed the increase of the thermal conductivity of heat sinks by introducing a graphite matrix instead of a metallic foam into the paraffin wax. 


\section{Modelling}

In this work, a Lattice Monte Carlo method is used to determine the effective thermal conductivity of cellular metals. In this method, the effective thermal conductivity is determined by evaluating the random walks of 'heat particles' that explore the geometry. The Lattice Monte Carlo method has already been successfully used to determine the thermal conductivities of many composite porous structures; see, for example, $[9,10]$.

The cellular metallic matrixes are represented by cubic-symmetrical model geometries. Fig. 1 shows one eighth of the unit cell of each structure. The voids in the models are occupied by the PCM paraffin. The Monte Carlo Lattices comprise the whole unit cell and periodic boundary conditions are defined on all surfaces of the unit cells. Accordingly, infinite structures without the influence of free surfaces are simulated. It should be mentioned here that Fig. 1 displays only schematic representations of the analysed geometries whose volume fractions $V_{\mathrm{M}}$ of the metallic matrix are not constant. The Monte Carlo lattices exhibit all the same volume fraction of the metallic matrix $V_{\mathrm{M}}=8 \%$. It has already been shown elsewhere (e.g. [11]) that the thermal conductivity of cellular metal increases with the volume fraction of the metallic matrix.

a)

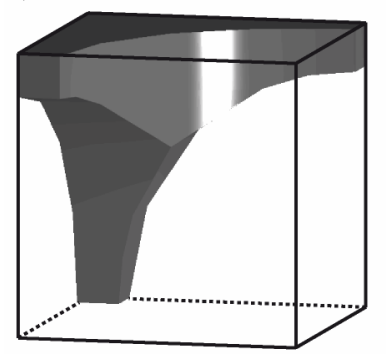

d)

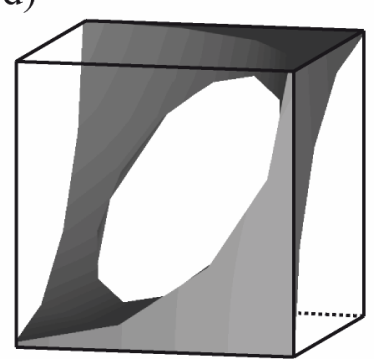

b)

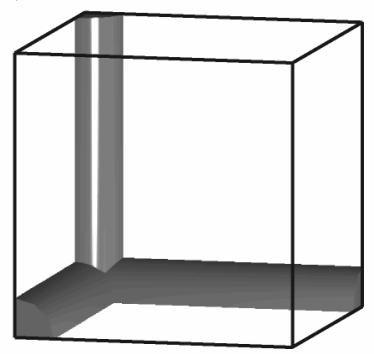

c)

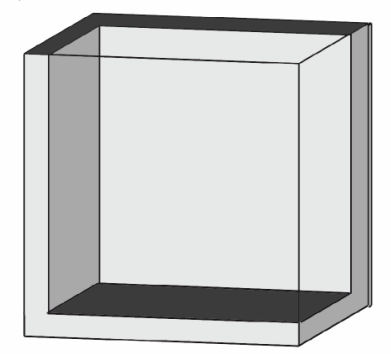

e)

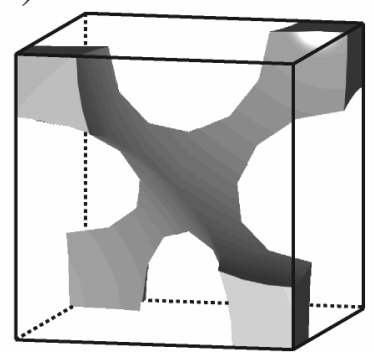

Fig. 1 Model geometries of cellular metals: a) perforated cube, b) orthogonal cylinders, c) closed cube, $d$ ) inverse b.c.c., e) inverse f.c.c.

Figure 1a) shows a perforated cube with cylindrical inclusions in the centre of each face of the unit cell. Its inverse structure is shown in Fig. 1b). Figure 1c) shows a closed-cell structure. This geometry is added for reasons of comparison; however, in a real application no PCM can be filled in such voids. Figures 1d) and 1e) are modelled by positioning spheres in a body-centred cubic (b.c.c.), respectively face-centred cubic (f.c.c.) arrangement in cubical unit cells and subtracting the volume of the spheres from the unit cell. Figure 2 shows that the complex geometry of an open-cell M-Pore ${ }^{\circledR}$ aluminium foam is best approximated by the inverse f.c.c. structure. 
a)

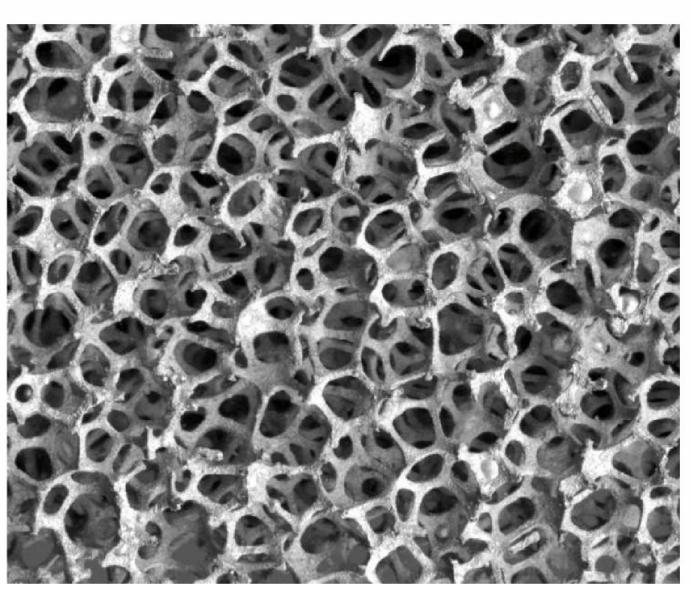

b)

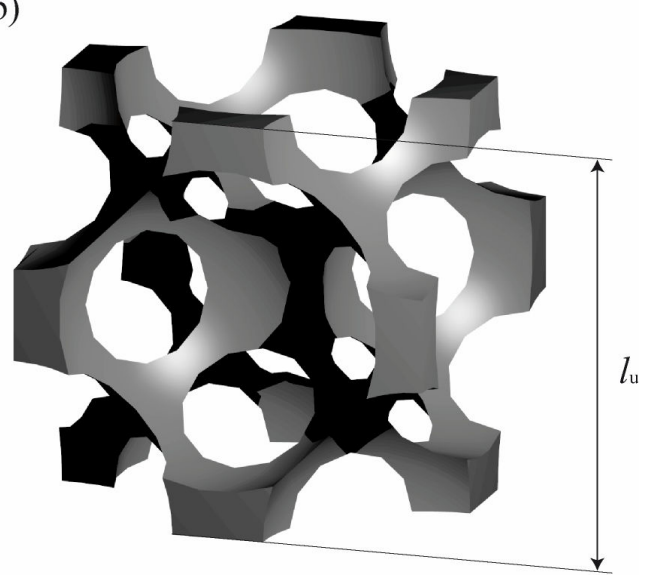

Fig 2 a) Open-cell M-Pore ${ }^{\circledR}$ aluminium foam, b) unit cell of inverse f.c.c. structure.

In order to investigate the enhancement of the thermal conductivity by diamond coating which can be applied e.g. by chemical vapor deposition (CVD)), a second set of Monte Carlo lattices is generated where a diamond coating with the constant thickness $t_{\mathrm{c}}=0.02 l_{\mathrm{u}}\left(l_{\mathrm{u}}\right.$ is the side length of the cubical unit cells) is deposited on the free surfaces of the models. Due to its closed porosity, the closed cube is not considered in these analyses. Since the area of the free surfaces varies with the geometry, also the volume fractions of the coatings change:

\begin{tabular}{lccc}
\hline Geometry & Metallic matrix & Coating & PCM \\
\hline Orthogonal cylinders & $8.0 \%$ & $3.4 \%$ & $88.6 \%$ \\
Perforated cube & $8.0 \%$ & $2.7 \%$ & $89.3 \%$ \\
Inverse bcc & $8.0 \%$ & $6.9 \%$ & $85.1 \%$ \\
Inverse fcc & $8.0 \%$ & $8.4 \%$ & $83.6 \%$ \\
\hline
\end{tabular}

Table $1 \quad$ Volume Fractions of coated model geometries.

Table 2 shows the thermal conductivities of the considered base materials. It can be observed that the two metals, i.e. aluminium and copper, exhibit a distinctly higher thermal conductivity than the phase change material paraffin. The thermal conductivity of the CVD diamond refers to coatings with a thickness between 0.3 and $0.6 \mathrm{~mm}$ and low concentrations of bonded hydrogen [13].

\begin{tabular}{cccc}
\hline $\begin{array}{c}\text { Aluminium }(\mathrm{Al}) \\
{[1]}\end{array}$ & $\begin{array}{c}\text { Copper }(\mathrm{Co}) \\
{[12]}\end{array}$ & $\begin{array}{c}\text { CVD diamond } \\
{[13]}\end{array}$ & $\begin{array}{c}\text { Paraffin }(\mathrm{PCM}) \\
{[1]}\end{array}$ \\
\hline $207 \mathrm{~W} /(\mathrm{m} \cdot \mathrm{K})$ & $380 \mathrm{~W} /(\mathrm{m} \cdot \mathrm{K})$ & $2100 \mathrm{~W} /(\mathrm{m} \cdot \mathrm{K})$ & $0.21 \mathrm{~W} /(\mathrm{m} \cdot \mathrm{K})$ \\
\hline
\end{tabular}

Table 2 Thermal conductivities of the base materials.

\section{Results and Discussion}

Figure 3 shows the result of the Lattice Monte Carlo analyses for the metal-PCM compounds. A strong dependence on the geometry can be found. Experimental data of the effective thermal conductivity of aluminium foams with similar volume fractions $\left(V_{\mathrm{M}} \approx 9 \%\right)$ is $6.7 \mathrm{~W} /(\mathrm{m} \cdot \mathrm{K})$ for air- 
filled structures and $7.6 \mathrm{~W} /(\mathrm{m} \cdot \mathrm{K})$ for water-filled structures [14]. These values lie approximately in the middle of the range of the results of the Lattice Monte Carlo analysis. The minimum effective thermal conductivities are obtained for the perforated cube (cf. Fig. 1 a)). A possible explanation is the metallic matrix concentrations in the corners of the unit cell which are connected only by thin rods. These thin rods act as bottlenecks to the thermal flow. Distinct material concentrations and relatively low $\lambda_{\text {eff }}$ can also be found in the inverse f.c.c. structure (cf. Fig. 1e)). In the case of the orthogonal cylinders (cf. Fig. 1b)), the thicknesses of the metallic rots are constant and a higher effective thermal conductivity is obtained. However, the material is located at the edges of the unit cell and 'heat particles' in its centre need to diffuse a long distance before they reach the high conductive metallic phase. The inverse bcc structure (cf. Fig. 1d)) shows no major material concentration and the material is distributed more homogeneously within the unit cell. Accordingly, this geometry exhibits the second highest thermal conductivity after the closed cube structure (cf. Fig. 1c)). An increase of approximately $80 \%$ of the effective thermal conductivity of the composite heat sinks can be achieved by substituting the aluminium matrix $\left(\lambda_{\mathrm{Al}}=207 \mathrm{~W} /(\mathrm{m} \cdot \mathrm{K})\right)$ by a copper foam $\left(\lambda_{\mathrm{Co}}=380 \mathrm{~W} /(\mathrm{m} \cdot \mathrm{K})\right)$.

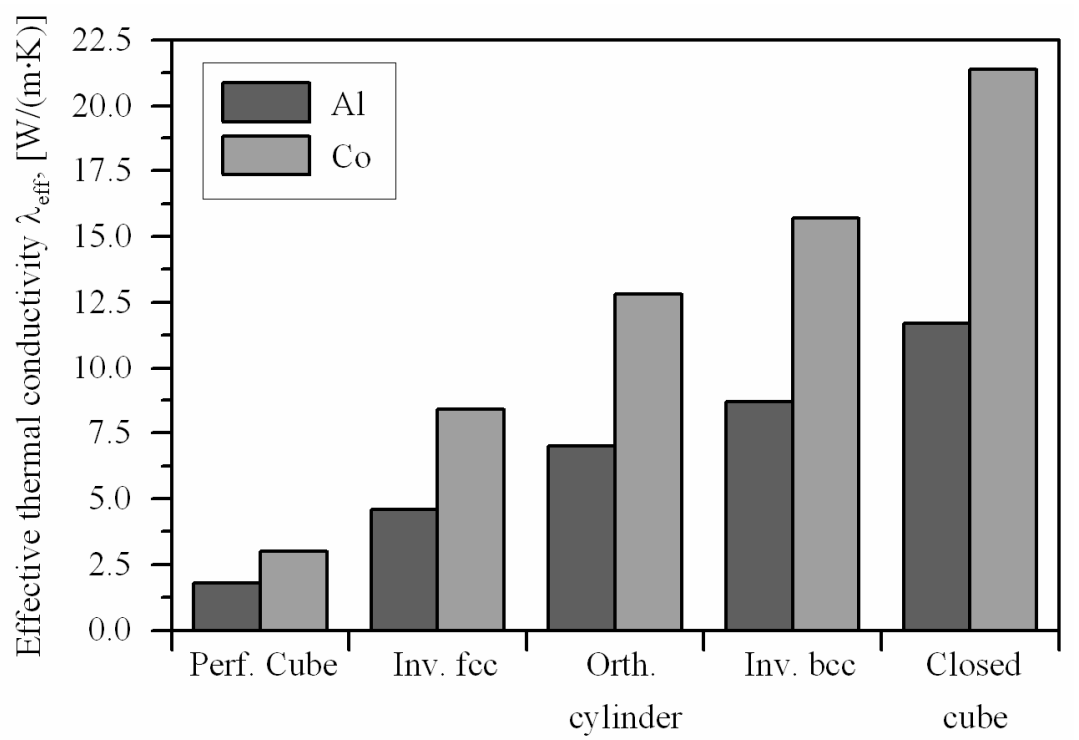

Figure 3 Effective thermal conductivities $\lambda_{\text {eff }}$ of metal-PCM composites.

Further increase of the effective thermal conductivity can be achieved by coating the cellular metallic matrixes with a highly thermal conducting CVD diamond film, cf. Table 3 . All considered geometries show a significant increase of $\lambda_{\text {eff. }}$ However, the effective thermal conductivity of the perforated cube and the orthogonal cylinders cannot reach the very high values of the inverse b.c.c. and inverse f.c.c. structures. The explanation is the varying volume fraction of the diamond coating (cf. Table 1). The maximum thermal conductivity is observed for the inverse f.c.c. structure which exhibits also the highest volume fraction of the coating (8.4\%).

\begin{tabular}{cccc}
\hline Orth. cylinders & Perf. cube & Inverse b.c.c. & Inverse f.c.c. \\
\hline 44 & 29 & 99 & 112
\end{tabular}

Table 3 Effective thermal conductivities of diamond coated copper-PCM composites in $W /(m \cdot K)$. 


\section{Conclusions}

Lattice Monte Carlo analyses on the effective thermal conductivity of paraffin based heat sinks were performed. A clear increase of the effective thermal conductivity of paraffin after adding an aluminium cellular matrix could be shown. This effect can be enhanced by substituting the aluminium with a copper alloy of higher thermal conductivity. Furthermore, a dramatic increase of the effective thermal conductivity was found for the simulation of CVD diamond coated copper matrixes. In addition to the dependence on the material composition, a distinct influence of the cellular model geometry was observed. The maximum effective thermal conductivity was found for the diamond coated f.c.c. structure which exceeds the value of pure paraffin by a factor of about 500 .

\section{Acknowledgements}

We wish to thank the Australian Research Council for its support of this work. T. Fiedler wishes to acknowledge the Portuguese Foundation of Science and Technology (FCT) for financial support.

\section{References}

[1] E.B.S. Mettawee and G.M.R. Assassa:, Sol. Energy, (2007) published online.

[2] A.G. De Jong and C.J. Hoogendoorn, in Thermal Storage of Solar Energy, edited by C. den OudenHague/Boston/London: TNO and Martinus Nijho, 1981. p. 12.

[3] I.M. Bugaje: Int. J. Energy Res. Vol. 21 (1997), p. 759.

[4] S.A. Khateeb, M.M. Farid, J.R. Selman and S. Al-Hallaj: J. Power Sources Vol. 128 (2004), p. 292.

[5] S.A. Khateeb, S. Amiruddin, M. Farid, J.R. Selman, S and Al-Hallaj: J. Power Sources Vol. 142 (2005), p. 345.

[6] S.T. Hong and D.R. Herling: Scripta Mater. Vol. 55 (2006), p. 887.

[7] T.K. Stovall and R.V. Arimilli, in: Proceedings of 23rd Intersociety Energy Conversion Engineering Conference, Denver, USA. 1988. p. 171.

[8] L.C. Chow, J.K. Zhong and J.E. Beam: Int. Comm. Heat Mass Transfer Vol. 23 (1996), p. 91.

[9] T. Fiedler, A. Öchsner, N. Muthubandara, I.V. Belova, G.E. Murch: Mater. Sci. Forum Vol. 553 (2007), p. 51.

[10] G. E. Murch and I. V. Belova, in Cellular and Porous Materials. Thermal Properties Simulation and Prediction, edited by A. Öchsner, G.E. Murch and J.S. de Lemos (Wiley $\mathrm{VCH}$; Weinheim) in press.

[11] T. Fiedler, E. Pesetskaya, A. Öchsner, J. Grácio: Mater. Sci. Forum Vol. 514 (2006), p. 754.

[12] J. Tian, T.J. Lu, H.P. Hodson, D.T. Queheillalt and H.N.G. Wadley: Int. J. Heat Mass Tran. Vol. 50 (2007) p. 2521.

[13] A.V. Sukhadolau, E.V. Ivakin, V.G. Ralchenko, A.V. Khomich, A.V. Vlasov and A.F. Popovich: Diam. Relat. Mater. Vol. 14 (2005) p. 589.

[14] V.V. Calmidi and R.L. Mahajan: ASME J. Heat Transfer. Vol. 121 (1999), p. 466. 